ARTICLE

\title{
Activity-dependent neuroprotective protein (ADNP) is an alcohol-responsive gene and negative regulator of alcohol consumption in female mice
}

\author{
Yarden Ziv $^{1,2}$, Nofar Rahamim ${ }^{2,3}$, Noa Lezmy ${ }^{2,3}$, Oren Even-Chen ${ }^{3}$, Ohad Shaham ${ }^{3}$, Anna Malishkevich ${ }^{1}$, Eliezer Giladi ${ }^{1}$, Ran Elkon ${ }^{1,2}$, \\ Illana Gozes (iD) ${ }^{1,2}$ and Segev Barak (iD ${ }^{2,3}$
}

Neuroadaptations in the brain reward system caused by excessive alcohol intake, lead to drinking escalation and alcohol use disorder phenotypes. Activity-dependent neuroprotective protein (ADNP) is crucial for brain development, and is implicated in neural plasticity in adulthood. Here, we discovered that alcohol exposure regulates Adnp expression in the mesolimbic system, and that Adnp keeps alcohol drinking in moderation, in a sex-dependent manner. Specifically, Sub-chronic alcohol treatment ( $2.5 \mathrm{~g} / \mathrm{kg} /$ day for 7 days) increased Adnp mRNA levels in the dorsal hippocampus in both sexes, and in the nucleus accumbens of female mice, $24 \mathrm{~h}$ after the last alcohol injection. Long-term voluntary consumption of excessive alcohol quantities ( 10-15 g/kg/ $24 \mathrm{~h}, 5$ weeks) increased Adnp mRNA in the hippocampus of male mice immediately after an alcohol-drinking session, but the level returned to baseline after $24 \mathrm{~h}$ of withdrawal. In contrast, excessive alcohol consumption in females led to long-lasting reduction in hippocampal Adnp expression. We further tested the regulatory role of Adnp in alcohol consumption, using the Adnp haploinsufficient mouse model. We found that Adnp haploinsufficient female mice showed higher alcohol consumption and preference, compared to Adnp intact females, whereas no genotype difference was observed in males. Importantly, daily intranasal administration of the ADNP-snippet drug candidate NAP normalized alcohol consumption in Adnp haploinsufficient females. Finally, female Adnp haploinsufficient mice showed a sharp increase in alcohol intake after abstinence, suggesting that Adnp protects against relapse in females. The current data suggest that ADNP is a potential novel biomarker and negative regulator of alcohol-drinking behaviors.

Neuropsychopharmacology (2019) 44:415-424; https://doi.org/10.1038/s41386-018-0132-7

\section{INTRODUCTION}

Alcohol use disorder (AUD) is a major worldwide health problem with concomitant medical, social and economic burdens, however available pharmacotherapy is very limited. It is believed that chronic excessive alcohol consumption may lead to neuroadaptations in the brain reward system, i.e., the mesolimbic dopaminergic system, which projects from the ventral tegmental area (VTA) to the nucleus accumbens (NAc), hippocampus and amygdala [1, 2]. For example, alcohol exposure leads to enhanced signaling in NAC of the phosphoinositide 3-kinase (PI3)-AKT and protein kinase A (PKA) pathways [3-6], as well as to alterations in the mesolimbic expression of growth factors [7-10] (for a review see [11]). These neuroadaptations, in turn, were postulated to promote AUD phenotypes $[1,2,12]$. Importantly, characteristic hallmark of AUD, such as loss of control over drinking, binge drinking episodes and withdrawal syndrome typically appear only after prolonged excessive alcohol consumption, suggesting that protective mechanisms may initially prevent or delay expression of the neuroadaptations underlying these phenotypes [11]. However, excessive exposure to alcohol may disrupt these protective mechanisms, leading to neuroadaptations and addiction phenotypes [11].

Activity-dependent neuroprotective protein (ADNP) is crucial for the early development of the brain [13], critical for axonal transport in adulthood [14], and has been implicated in dendritic spine formation and synapse plasticity [15]. ADNP also functions as a transcription factor as part of the chromatin remodeling complex SWItch/Sucrose Non-Fermentable (SWI/SNF). As such, ADNP is crucial for the regulation of transcription of $>400$ genes during development [16-18] and in the adult brain in a sex-dependent manner [14, 19]. ADNP abnormalities have been implicated in several neurodegenerative and neuropsychiatric diseases, including Alzheimer's disease [20], schizophrenia [21, 22], and autism [23, 24]. Partial deficiency in mouse Adnp results in altered gene regulation, axonal transport deficits, cognitive and social deficits and culminates in age-related neuronal demise [14, 19, 25].

Studies on fetal alcohol syndrome showed that alcohol consumption by pregnant mice causes an increase in Adnp expression in the embryonic brain even 10 days after alcohol administration, implicating ADNP expression as a protector of the forming brain from alcohol damage [26]. Furthermore, impaired

\footnotetext{
${ }^{1}$ Department of Human Molecular Genetics and Biochemistry, Sackler Faculty of Medicine, Tel Aviv University, 69978 Tel Aviv, Israel; ${ }^{2}$ Sagol School of Neuroscience and Adams Super Center for Brain Studies, Tel Aviv University, 69978 Tel Aviv, Israel and ${ }^{3}$ School of Psychological Sciences, Tel Aviv University, 69978 Tel Aviv, Israel Correspondence: Illana Gozes (igozes@post.tau.ac.il) or Segev Barak (barakseg@post.tau.ac.il)

These authors contributed equally: Illana Gozes, Segev Barak
}

Received: 25 June 2017 Revised: 20 June 2018 Accepted: 21 June 2018 Published online: 27 June 2018 
ADNP synthesis and release was observed in astroglial cell cultures from prenatal alcohol-exposed rat foetuses [27] and transcriptome organization analysis linked ADNP to chronic alcohol abuse in the human brain [28].

To elucidate the role of ADNP expression and activity in alcohol-drinking behaviors in the adult brain, we determined whether alcohol exposure affects Adnp expression in the mesolimbic system, and utilized the Adnp-deficient mouse model to investigate the regulatory role of ADNP in alcohol consumption and relapse.

\section{MATERIALS AND METHODS}

See Supplemental Information for details on reagents, standard qRT-PCR and alcohol administration.

\section{Animals}

All protocols conformed the guidelines of the Institutional Animal Care and Use Committee of Tel Aviv University and the Israeli Ministry of Health, as well as the guidelines of the NIH (animal welfare assurance number A5010-01). All efforts were made to minimize the number of animals used. All animals were housed in Tel-Aviv University Animal Facility (12-h light-dark cycle-lights on at 4 a.m., food and water ad libitium). C57BL/6J mice (25-30 g) were housed 3-4/cage; $A d n p^{+/+}$and littermates, $A d n p^{+/-}$mice (outbred with ICR strain for 30 generations in Tel-Aviv University Animal Facility; 25-30 g) [13, 14, 19, 25] were individually housed. Bedding was standard sterilized dust-saw, and rodent chow used were Teklad Global 18\% protein (Envigo, Israel).

Tissue collection

Mice were euthanized, and brains were quickly removed and placed on an ice-cold platform. Tissues from the NAc, dorsal hippocampus and VTA were carefully dissected from 1-mm slices using a brain matrix and immediately snap-frozen in liquid nitrogen and stored at $-80^{\circ} \mathrm{C}$ until use for further analysis for qRT-PCR, as previously described [7, 29].

\section{Alcohol/saccharin/quinine-consumption procedures}

Two-bottle choice training began after 1 week of habituation for individual housing. The position (left or right) of each solution was alternated as a control for side preference. Consumption levels were normalized to body weight.

Continuous access to escalating concentrations of alcohol, saccharin or quinine in two-bottle choice. Mice had access to one bottle containing tap water, while the other contained an increasing concentration of alcohol $(3 \%, 6 \%, 10 \%$, and $20 \%, \mathrm{v} / \mathrm{v})$ [30], saccharin $(0.003 \%, 0.01 \%, 0.03 \% \mathrm{w} / \mathrm{v})$ [7], or quinine $(0.03,0.06 \mathrm{mM})[30,31]$, with 7 days of access for each concentration. Fresh fluids were provided each time the concentration was changed.

Intermittent access to $20 \%$ alcohol in two-bottle choice (IA2BC). Mice were trained to consume alcohol in the IA2BC procedure as previously described $[4,32,33]$. Briefly, mice received three $24-\mathrm{h}$ sessions of free access to two-bottle choice per week (water and $20 \%$ alcohol v/v) on Sundays, Tuesdays, and Thursdays, with 24 or $48 \mathrm{~h}$ of alcohol-deprivation periods in between the alcohol-drinking sessions. During the withdrawal periods, animals received only water. In the Adnp expression experiment, control mice received access to two water bottles. Water and alcohol bottles were weighed before and after each alcohol-drinking session. In the IA2BC experiment with Adnp ${ }^{+/-}$mice, after 15 alcohol-access sessions, mice underwent a 12-day alcohol-deprivation period, after which they received three additional intermittent access to alcohol sessions.

Continuous access to $10 \%$ alcohol in two-bottle choice and administration of NAP (NAPVSIPQ). Mice had access to one bottle containing tap water, and one bottle containing $10 \%$ alcohol $(\mathrm{v} / \mathrm{v})$ $[34,35]$ for 4 weeks. Water and alcohol bottles were weighed every $24 \mathrm{~h}$ to measure fluid intake. Mice were habituated for the intranasal administration procedure for a week prior to NAP or vehicle administration (week 1, "no treatment week"). Intranasal administration was performed by gently restraining the mice, and placing a pipet tip near their nostrils. NAP was dissolved in a solution, in which each milliliter included $7.5 \mathrm{mg}$ of $\mathrm{NaCl}$, $1.7 \mathrm{mg}$ of citric acid monohydrate, $3 \mathrm{mg}$ of disodium phosphate dihydrate, and $0.2 \mathrm{mg}$ of benzalkonium chloride solution (50\%), as previously described [36]. The half-life of NAP in the plasma after inranasal administration is $\sim 70 \mathrm{~min}$ in rats [37]. Vehicle was administered intranasally on week 2 . NAP $(0.5 \mu \mathrm{g} / 5 \mu \mathrm{l})$, the ADNPderived neuroprotective peptide (drug candidate) [38, 39], was administered intranasally [40] on weeks 3 and 4, once a day, 5 days/week (Sunday-Thursday), at 11:00 am.

Comparative analysis of expression data from $\mathrm{Adnp}^{+/-}$mice and alcohol-treated mice

Hippocampal RNA-seq expression data (count matrix) of $A d n p^{+/+} /$ Adn ${ }^{+/-}, 1 / 5$-month old, male and female mice [14] [Gene Expression Omnibus (GEO) database (accession number: GSE72664)] was utilized. Data processing was performed separately for each sex and age group (i.e., 1-month old females, 5month old males), using the statistical computing environment $R$ (http://www.r-project.org/). Genes that failed to produce 20 reads or more in at least two samples were omitted. Quantile normalization was performed on the remaining genes. For each gene, mean values were calculated for each genotype, and were subjected to a second round of quantile normalization. Finally, fold-change (FC) values of $A d n p^{+/-}$in relation to $A d n p^{+/+}$were calculated. To avoid inflation of high FC values for minimally expressed genes, values $<10$ th percentile, were set into that value. The sets of the top 250 up-regulated and top 250 down-regulated genes were defined as "Adnp ${ }^{+/-}$up-signature" and "Adnp ${ }^{+/-}$ down-signature", respectively. Distinct "Adnp ${ }^{+-}$up" and "Adnp ${ }^{+-}$ down" signatures were defined for each combination of sex + age (Supplemental Table S1).

Affymetrix array data of hippocampal gene expression in male mice that were chronically exposed to alcohol [41] was obtained from GEO database (accession number: GSE72517). The analyzed data consisted of samples from adult male mice that underwent chronic intermittent exposure to vaporized alcohol in inhalation chambers and control mice that received plain air in the chambers, with tissues harvesting $\mathrm{O} \mathrm{h}$ after the completion of the treatment. The data was processed within $\mathrm{R}$ programming environment. Quantile normalization was applied on all probes and the value of the 25th percentile was set as floor value (higher percentile level was applied here because expression array data is nosier than RNAseq for minimally expressed genes). After calculating the FC values of the chronic alcohol exposure treatment in relation to the control, "chronic alcohol up-signature" and "chronic alcohol down-signature" were defined as described above.

The significance of the overlap between the $\mathrm{Adnp}^{+/-}$signatures (up, down and up and down combined) and the chronic alcohol signatures were tested using Fisher's exact test. Association between the direction of the "response" (up/down) to alcohol and Adnp haploinsufficiency was tested using Pearson's chi square test.

Identification of enriched gene ontology (GO) biological process categories in each group of overlapping genes was performed using the TANGO tool from EXPANDER [42]. Categories with $p<0.05$ after correcting for multiple testing, were considered significant.

Statistical analysis

Adnp expression was normalized to Gapdh expression [7, 8, 29]. Importantly, we recently showed that Gapdh levels are not affected in mice under the alcohol drinking protocols used here [7]. Expression in each brain region was further normalized to the 
control group (saline-treated or water-treated group), for each sex separately. Expression data were analyzed by one-way ANOVA as indicated. Alcohol and saccharin consumption and preference data were analyzed by a mixed-model ANOVA with betweensubjects factors of sex (male, female) and genotype (Adnp ${ }^{+/+}$, $A d n p^{+/-}$) and a repeated-measures factor of sessions. LSD post hoc analysis followed significant effects.

\section{RESULTS}

Short exposure to alcohol increases Adnp mRNA expression in the NAc and dorsal hippocampus

First, we tested whether a short-term, controlled exposure to alcohol would affect Adnp expression in the mesolimbic brain regions, NAc, VTA, and dorsal hippocampus. C57BL/6J mice were injected with alcohol $(2.5 \mathrm{~g} / \mathrm{kg}$ in a $20 \%$ alcohol v/v solution, i.p.) or saline once daily for 7 days, and were sacrificed 2 or $24 \mathrm{~h}$ after the last alcohol injection. As shown in Fig. 1, we found that this sub-chronic alcohol treatment increased Adnp mRNA levels in the dorsal hippocampus $24 \mathrm{~h}$, but not $2 \mathrm{~h}$ after the last alcohol injection, in both male and female mice (one-way ANOVA; males: $F_{(2,10)}=5.94, p<0.05$; post-hoc, $p<0.02$. Females: $F_{(2,10)}=5.22$,
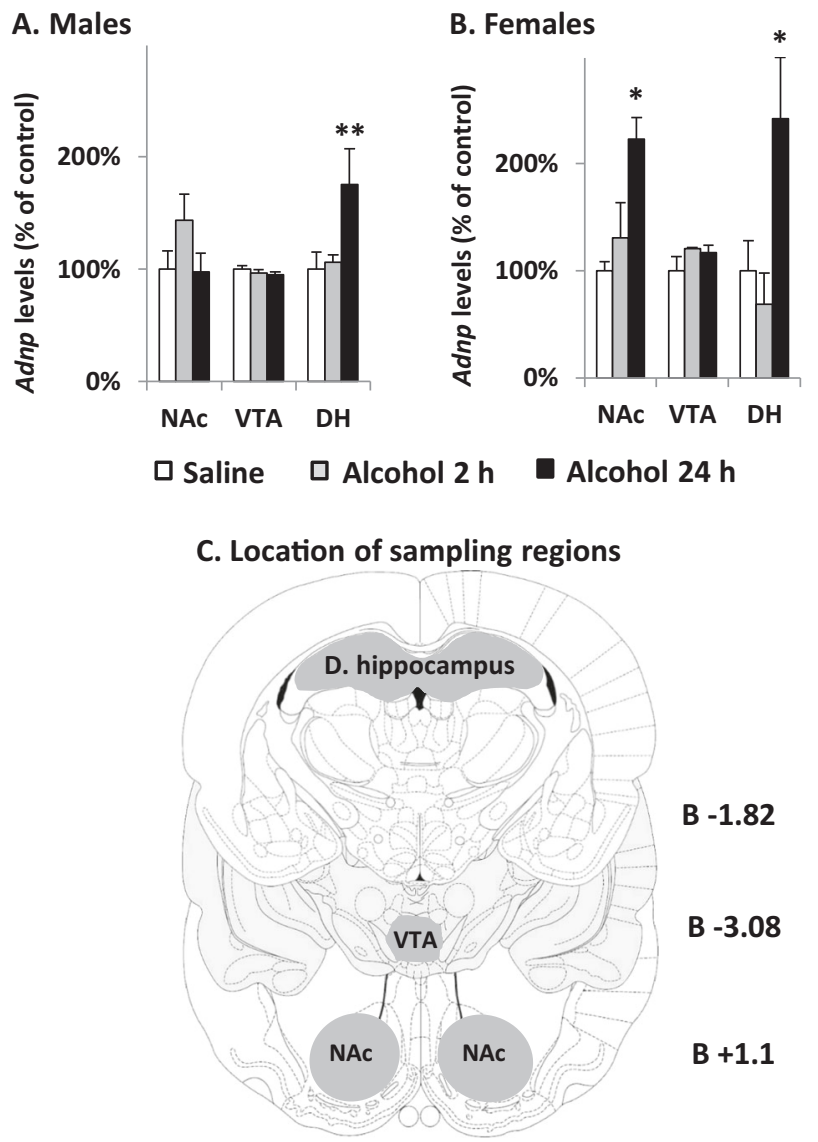

Fig. 1 Alcohol exposure alters Adnp mRNA expression in the nucleus accumbens (NAC) and dorsal hippocampus (DH). C57BL/6J mice were injected with alcohol $(2.5 \mathrm{~g} / \mathrm{kg}, 20 \% \mathrm{v} / \mathrm{v}$, i.p.) or saline for 7 consecutive days. Brain tissues were collected $2 \mathrm{~h}$ (Alcohol $2 \mathrm{~h}$ ) or $24 \mathrm{~h}$ (Alcohol $24 \mathrm{~h}$ ) after the last alcohol injection. a, b mRNA expression of Adnp in male (a) and female (b) mice. Transcripts (mRNA) contents were determined by quantitative reverse transcriptase polymerase chain reaction (qRT-PCR) and normalized to Gapdh. Data are means \pm SEM. Each brain region was normalized to its saline control. c Schematic representation of the sampling regions. $n=6-10$ per group. ${ }^{*} p<0.05,{ }^{* *} p<0.01$ $p<0.05$, post hoc, $p<0.05)$. In addition, Adnp expression was increased at the $24 \mathrm{~h}$ time point in the NAc of female, but not male mouse (one-way ANOVA; females: $F_{(2,9)}=10.87, p<0.005$; post hoc: $p<0.005$; males: $p>0.05)$. We found no changes in the expression of Adnp in the VTA ( $p^{\prime} s>0.05$ ).

Thus, short alcohol exposure increased Adnp expression in the mouse NAc and dorsal hippocampus in a sex-dependent manner.

Long-term voluntary consumption of excessive alcohol levels leads to reduced Adnp expression selectively in female mice Next, we determined the effects of long-term voluntary alcohol consumption on the expression of Adnp. C57BL/6J Mice were trained to consume alcohol in the intermittent access to $20 \%$ alcohol in two-bottle choice procedure [4, 33] for 5 weeks (Fig. 2a). In this drinking protocol, mice consume high quantities of alcohol, typically with an average alcohol intake of $10-15 \mathrm{~g} / \mathrm{kg} / 24 \mathrm{~h}$, which generates blood alcohol concentration (BAC) of $>100 \mathrm{mg} \%$, and alcohol preference of $\sim 50 \%[34,43]$. The NAc, VTA, and dorsal hippocampus of mice were collected at the end of a 24-h alcohol drinking session (mean alcohol consumption: males, $\sim 10 \pm 1.5 \mathrm{~g} /$ $\mathrm{kg} / 24 \mathrm{~h}$, alcohol preference $\sim 50 \pm 3.5 \%$; females $\sim 13 \pm 3.5 \mathrm{~g} / \mathrm{kg} / 24$ h, alcohol preference $\sim 4 \pm 6 \%$ ), or after a 24 -h alcohol withdrawal period. Control mice consumed only water.

We found that among male mice, Adnp mRNA levels were increased in the dorsal hippocampus after a $24 \mathrm{~h}$ alcohol drinking session, but the level returned to baseline (water-drinking controls) after $24 \mathrm{~h}$ of withdrawal (Fig. 2b; one-way ANOVA: $F_{(2,13)}=5.32, p<0.05$, post hoc comparisons, $\left.p^{\prime} s<0.05\right)$. However, in female mice, the expression of Adnp was reduced after a 24-h alcohol-drinking session, and this reduction persisted after a 24-h withdrawal (Fig. 2c; one-way ANOVA: $F_{(2,13)}=7.53, p<0.01$, post hoc comparisons, $\left.p^{\prime} s<0.05\right)$. No changes in Adnp levels were detected in the VTA or NAc $\left(p^{\prime} s>0.05\right)$. As the male and female experiments were performed separately (Figs. 1 and 2), we also conducted a direct comparison of Adnp expression in the dorsal hippocampus and found similar expression in the naïve male and female mice (Supplemental Fig. S1).

Together, these results indicate that long-term excessive consumption of alcohol leads to differential alterations in hippocampal Adnp expression: a transient increase in males, and a sustained decrease in females.

Haploinsufficiency of Adnp leads to increased alcohol, but not saccharin or quinine intake, selectively in female mice

As we found that the Adnp gene responds to alcohol in a sexdependent manner, we further tested whether the protein plays a role in the development and/maintenance of alcohol consumption. To this end, we used Adnp haploinsufficient mice (Adnp ${ }^{+/-}$ $[14,19,25])$, as ADNP is crucial for brain formation and knockout mice die during embryonic development [13]. Originally, a reduction of $42 \%$ in the cortex, of $38 \%$ in cerebellum, and of $50 \%$ in hippocampus was observed in the levels of Adnp mRNA in Adnp ${ }^{+/-}$mice compared with Adnp intact mice [25].

To elucidate the effects of Adnp haploinsufficiency on alcohol drinking, we compared voluntary alcohol consumption between the $\mathrm{Adnp}^{+/-}$and $\mathrm{Adnp^{+/+ }}$ mice in a two-bottle choice paradigm (alcohol vs. water). Thus, mice had continuous access to a bottle of water and a bottle containing escalating concentrations of alcohol (3\%, 6\%, 10\%, 20\%) [30]. Alcohol concentration was increased every week.

Figure 3 presents the average daily consumption and preference levels for each of these alcohol concentrations, among male and female $A d n p^{+/-}$and $A d n p^{+/+}$littermates. We found that female mice showed considerably higher alcohol consumption and preference compared to males, regardless of genotype or alcohol concentrations. Importantly, $A d n p^{+/-}$females showed higher alcohol consumption and preference, compared to female $\mathrm{Adnp}^{+/+}$controls, whereas no difference was observed in males. 


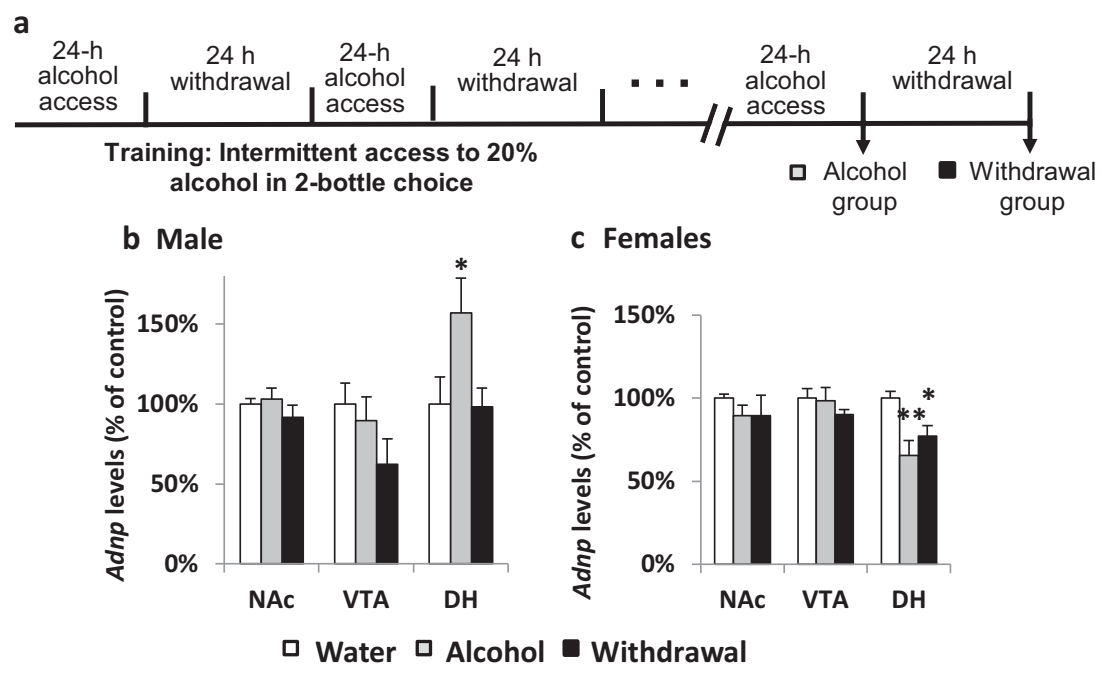

Fig. 2 Voluntary alcohol consumption leads to sex-dependent alterations of Adnp expression in the dorsal hippocampus (DH). C57BL/6J mice consumed alcohol in the intermittent access to $20 \%$ alcohol in two-bottle choice procedure for 5 weeks. Control mice consumed water only. Tissues were collected immediately at the end of the last 24-h drinking session (Alcohol group) or $24 \mathrm{~h}$ after the termination of the last drinking session (Withdrawal group). a Experimental timeline scheme. b, c Adnp mRNA levels in male (b) and female (c) mice were determined by quantitative reverse transcriptase polymerase chain reaction (qRT-PCR), and normalized to Gapdh. Data are means \pm SEM. Each brain region was normalized to its water control. $n=8-9$ per group. ${ }^{*} p<0.05,{ }^{* *} p<0.01$

Total fluid intake remained constant, suggesting that the effect in females did not stem from a general consummatory difference between genotypes.

Three-way mixed model ANOVA revealed significant main effects of sex (consumption: $F_{(1,29)}=36.85$; preference: $F_{(1,29)}=$ 19.72 , $\left.p^{\prime} s<0.0005\right)$, genotype (consumption: $F_{(1,29)}=10.69$; preference: $\left.F_{(1,29)}=9.84, p^{\prime} s<0.005\right)$, and alcohol concentration (consumption: $F_{(3,87)}=36.40$; preference: $F_{(3,87)}=20.48, \quad p^{\prime} s<$ $0.0001)$, as well as a significant sex $\times$ genotype interaction (consumption: $F_{(1,29)}=9.34$; preference: $F_{(1,29)}=6.68, p^{\prime} s<0.02$ ), and sex $\times$ concentration (consumption: $F_{(3,87)}=15.95$; preference: $\left.F_{(3,87)}=5.53, p^{\prime} s<0.005\right)$, genotype $\times$ concentration (consumption: $F_{(3,87)}=2.88$; preference: $\left.F_{(3,87)}=4.83, p^{\prime} s<0.05\right)$ and a sex $x$ genotype $x$ concentration interaction (consumption: marginally significant, $F_{(3,87)}=2.40, p=0.073$; preference: $F_{(3,87)}=3.90, p<$ $0.05)$. No interaction effects were found in water or total fluid consumption. Significant post hoc comparisons between genotypes are marked by asterisks in Fig. 3. Together, our results indicate that reduced Adnp gene dosage leads to increased alcohol intake in female mice.

Next, we tested whether the effect of Adnp haploinsufficiency is specific for alcohol. Therefore, we tested consumption of a sweet, non-drug reward, saccharin $(0.005 \%, 0.01 \%$, and $0.03 \%)$, and of a bitter, aversive substance, quinine $(0.03,0.06 \mathrm{nM})$, in a continuous access two-bottle choice protocol similar to the one used for alcohol intake. As we found that the effect on alcohol drinking was not observed in males, we included only females in the follow-up saccharin and quinine experiments. As shown in Fig. $3 e, f$, we found no difference between genotypes in saccharin or quinine intake, suggesting that the effect of Adnp deficiency is specific to alcohol, and does not apply to sweet reinforcers or bitter-taste solutions. Two-way mixed model ANOVA revealed a significant main effect of saccharin concentration $\left(F_{(2,34)}=87.93, p<0.0001\right)$, and of quinine concentration $\left(F_{(1,12)}=5.73, p<0.05\right)$, but no effects of genotype and no interaction effects ( $\left.p^{\prime} s>0.05\right)$.

Together, our results indicate that reduced Adnp expression in female mice leads to increased consumption of alcohol, but not of a sweet, non-drug reinforcer, or of a bitter solution.
NAP reduces excessive alcohol intake in female Adnp haploinsufficient mice and rescues the wild type phenotype As female $A d n p^{+/-}$mice showed higher levels of alcohol intake compared to $\mathrm{Adnp}^{+/+}$controls in the continuous access drinking paradigm, we next set out to rescue the wild type phenotype by restoring the Adnp function in these animals. Thus, we tested whether the effect of Adnp haploinsufficiency on alcohol intake could be reversed by intranasal administration of NAP (NAPVSIPQ), a neuroprotective peptide derived from Adnp [39, 44]. NAP has been shown to ameliorate Adnp deficiencies in the haploinsufficient mouse model [25], mechanistically by enhancing ADNP association with its microtubule and autophagy targets [15, 22, 40,44-46]. As we did not find an effect of Adnp genotype in male mice, we included only females in this experiment.

$\mathrm{AdnP}^{+/-}$and $\mathrm{Adnp^{+/+ }}$ mice were given continuous access to two bottles: a bottle of water and a bottle of $10 \%$ alcohol, for 4 weeks. After one week of drinking without treatment, all mice received vehicle treatment for one week, followed by intranasal NAP $(0.5 \mu \mathrm{g} / 5 \mu \mathrm{l})$ treatment for 2 weeks (Fig. 4a). As Fig. $4 \mathrm{~b}$ depicts, over the first 2 weeks of drinking (no treatment or intranasal vehicle treatment) $A d n p^{+/-}$females showed higher alcohol consumption as compared to their Adnp ${ }^{+/+}$littermates, replicating our results above. Strikingly, NAP treatment reduced alcohol consumption in Adnp+/- mice, which did not differ from $A_{d n p^{+/+}}$controls. We found no effect of NAP on alcohol intake in the $A d n p^{+/+}$females.

Two-way mixed model ANOVA revealed a significant main effect of treatment $(F(3,81)=6.05, p<0.001)$, as well as a significant treatment $\times$ genotype interaction $(F(3,81)=8.66, p<$ $0.0001)$. Post hoc analysis: significant differences between genotypes during the no treatment $(p<0.05)$ and vehicle $(p<$ 0.01 ) periods, and significant differences in $A d n p^{+/-}$mice between NAP treatment and no treatment or vehicle treatment $\left(p^{\prime} s<\right.$ $0.001)$.

These results indicate that restoration of ADNP function by NAP administration rescues the wild type phenotype of the $\mathrm{Adnp}^{+/-}$ mice, by reversing the abnormal increase in alcohol intake seen in Adnp haploinsufficient female mice. 

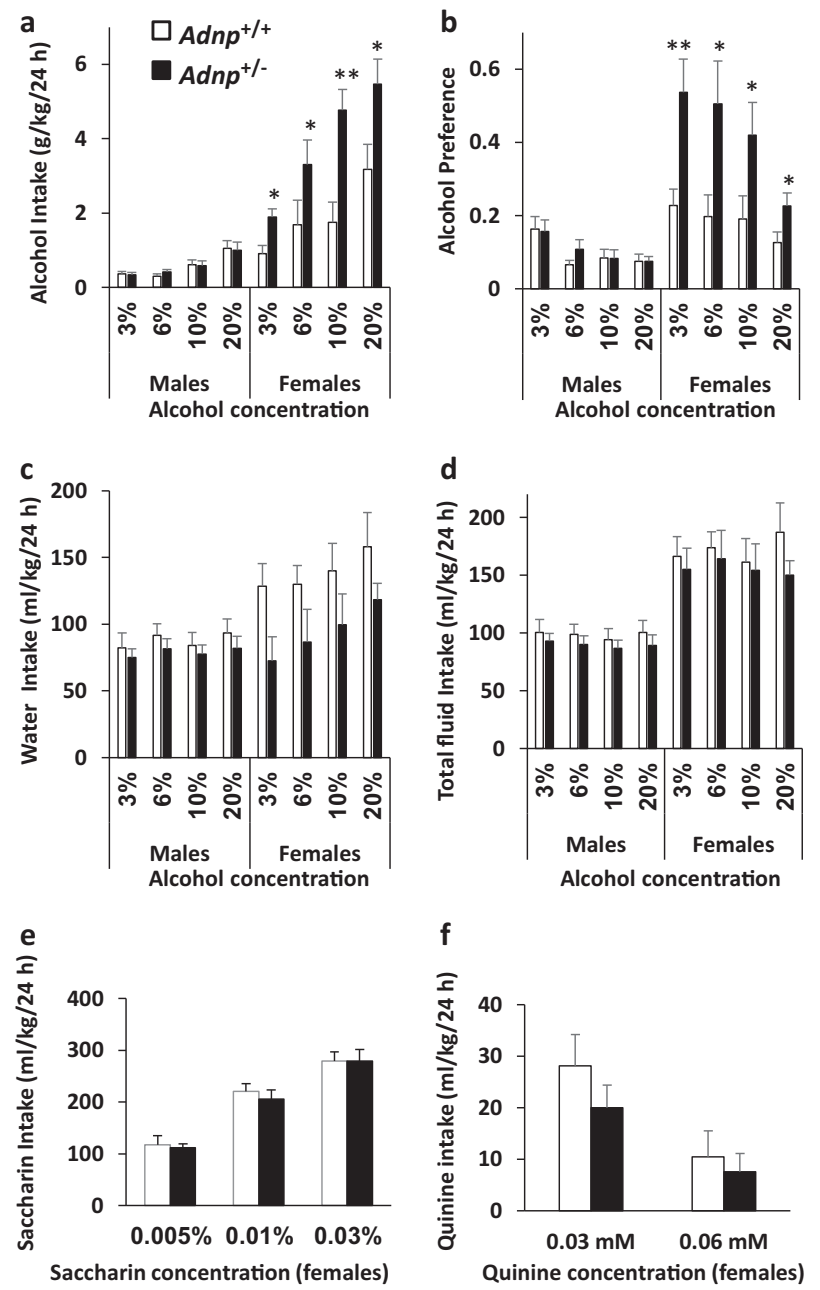

Fig. 3 Adnp haploinsuffiency leads to increased voluntary alcohol consumption and preference in female mice. Adnp ${ }^{+/-}$or $A d n p^{+/+}$ mice received continuous access to increasing concentration of alcohol (3\%,6\%, 10\%, 20\% v/v, a week for each concentration) concomitantly with water access (two-bottle choice). a Amount of alcohol $(\mathrm{g} / \mathrm{kg}$ ) consumed. b Preference to alcohol [(alcohol intake/ (alcohol + water intake)]. c Water intake $(\mathrm{ml} / \mathrm{kg})$. d Total fluid intake (alcohol + water; $\mathrm{ml} / \mathrm{kg}$ ). e $A d n p^{+/-}$or $A d n p^{+/+}$mice received continuous access to increasing concentration of saccharin $(0.003 \%, 0.01 \%, 0.03 \% \mathrm{w} / \mathrm{v}$, a week for each concentration) concomitantly with water access (two-bottle choice). f $\mathrm{Adnp}^{+/-}$or $A d n p^{+/+}$mice received continuous access to increasing concentration of quinine $(0.03,0.06 \mathrm{mM}$, a week for each concentration) concomitantly with water access (two-bottle choice). Data are means \pm SEM. $n=8-10$ per group. ${ }^{*} p<0.05$

Haploinsufficiency of the Adnp gene leads to increased alcohol intake after a period of abstinence in female mice

As we found that Adnp haploinsufficiency led to increased alcohol intake in the escalating alcohol concentration drinking protocol, we next tested whether these mice will show increased transition to excessive alcohol intake, in another model of drinking escalation, namely, the intermittent access to $20 \%$ alcohol in two-bottle choice. In this model, alcohol concentration is kept constant throughout training, however, training consists of repeated cycles of alcohol drinking and withdrawal, which typically lead to excessive alcohol drinking $[33,34]$. Given that the genetic background of the Adn $p^{+/-}$mice $[14,19]$ was mixed with ICR strain, which consumes low levels of alcohol $[47,48]$, we expected alcohol intake to be moderate. a

\begin{tabular}{|c|c|}
\hline $\begin{array}{c}\text { No } \\
\text { treatment } \\
(1 \text { week })\end{array}$ & $\begin{array}{l}\text { Vehicle } \\
\text { treatment } \\
(1 \text { week })\end{array}$ \\
\hline
\end{tabular}

Continuous access to $10 \%$ alcohol in 2-bottle choice

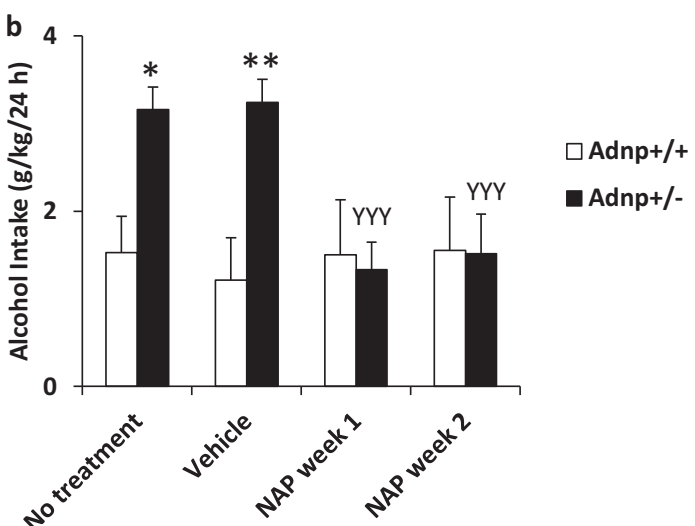

Fig. 4 NAP treatment reverses the increases in alcohol consumption in Adnp haploinsufficient female mice. Adnp $p^{+/-}$or $A d n p^{+/+}$mice consumed alcohol in the continuous access to $10 \%$ alcohol in twobottle choice procedure for 4 weeks. On week 2 they were treated with vehicle, and on weeks 3-4 they were treated with intranasal $\operatorname{NAP}(0.5 \mu \mathrm{g} / 5 \mu \mathrm{l}), 5$ days per week. a Experimental timeline scheme. b Daily alcohol intake, averaged by weeks of treatment. Data are

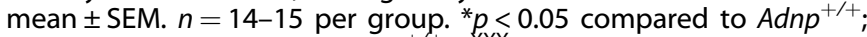
${ }^{* *} p<0.01$ compared to $A d n p^{+/+} ;{ }^{Y Y Y} p<0.001$ compared to no treatment and vehicle

Mice received concomitant access to a bottle of water and a bottle of $20 \%$ alcohol for $24 \mathrm{~h}$ sessions three days a week (Sunday, Tuesday, Thursday), with 24-h or 48-h sessions of alcohol withdrawal and only water access. We found that, mice increased their alcohol intake and preference as a factor of training sessions, and that female mice consumed more alcohol compared to males and showed sharper escalation in alcohol intake. However, we found no difference between genotypes (Fig. 5a). A mixed model three-way ANOVA for both consumption and preference yielded main effects of sex $\left(F_{(1,23)}=13.53, p<0.002\right)$ and sessions $\left(F_{(14,322)}\right.$ $=7.60, p<0.0001)$ and a sex $\times$ sessions interaction $\left(F_{(14,322)}=2.56\right.$, $p<0.002)$.

As we did not find a genotype effect on drinking of $20 \%$ alcohol in this drinking protocol, we next tested whether relapse to alcohol drinking after a period of abstinence would be affected by Adnp deficiency. Thus, mice were deprived from alcohol for 12 days (water was given ad libitum), followed by tests for relapse to alcohol consumption for 3 days.

Figure $5 \mathrm{~b}$ depicts the alcohol intake before and after the abstinence period. We found that while there was no difference between pre-abstinence and post-abstinence alcohol intake among $A d n p^{+/+}$female mice, female $A d n p^{+/-}$mice relapsed to higher alcohol consumption after abstinence, compared to their pre-abstinence consumption. No difference was found among males.

A mixed model three-way ANOVA yielded a main effect of sex $\left(F_{(1,33)}=29.47, p<0.0001\right)$, as well as significant interactions of sex $\times$ genotype $\left(F_{(1,33)}=4.35, \quad p<0.05\right)$, abstinence $\times$ genotype $\left(F_{(1,33)}=7.29, p<0.02\right)$, and sex $\times$ genotype $\times$ abstinence $\left(F_{(1,33)}\right.$ $=6.01, p<0.02)$. Post hoc analysis: a significant difference between pre-abstinence and post-abstinence alcohol intake in female $A d n p^{+/-}$mice $(p<0.001)$, but not in any of the other conditions $(p>0.05)$.

These results indicate that Adnp haploinsufficient female mice show increased alcohol relapse after prolonged consumption of high levels of alcohol. 


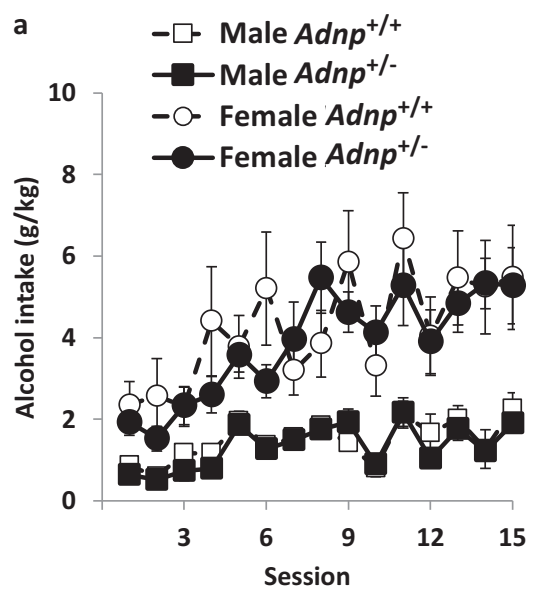

b

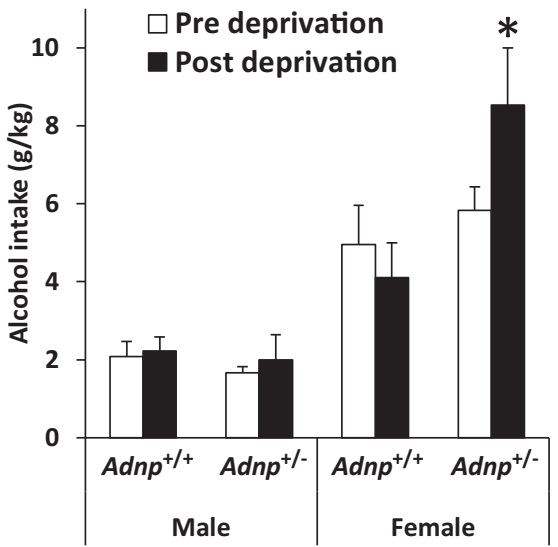

Fig. 5 Adnp haploinsufficiency increases relapse to alcohol drinking after a period of abstinence in female mice. Adnp ${ }^{+/-}$or $A d n p^{+/+}$mice received access to $20 \%$ alcohol 3 days a week for 5 weeks in the intermittent access to $20 \%$ alcohol in two-bottle choice (IA2BC) procedure. Mice were then subjected to 12 days of alcohol abstinence in the home cage. Post-abstinence alcohol intake was measured for three sessions (intermittent days) after the deprivation period. a Amount of alcohol $(\mathrm{g} / \mathrm{kg})$ consumed during the IA2BC training. $\mathbf{b}$ Average alcohol intake in the three sessions before and after the abstinence period. $n=8-10$ per group. ${ }^{*} p<0.05$

Adnp haploinsufficiency regulates hippocampal alcohol-associated genes in a sex-dependent manner

We observed sex-dependent alteration in Adnp expression in response to alcohol exposure, as well as sex-dependent effect of Adnp haploinsufficiency on alcohol consumption. To further elucidate the sex-dependency of the Adnp-alcohol interactions, we applied a comparative analysis of hippocampal expression data (RNA-seq) from naïve male/female, 1-month and 5-month old $\mathrm{Adnp}^{+/+}$or Adnp ${ }^{+/-}$mice [14], with microarray results obtained in male mice, subjected to chronic alcohol exposure [41].

An "Adnp ${ }^{+/-}$up signature" and "Adnp ${ }^{+/-}$down signature", containing the top 250 up/down regulated genes for each sex, were compared with the up/down signature from the chronicalcohol exposure mouse expression data. First, for each sex separately, we examined the overlap between the 500 genes comprising the $A d n p^{+/-}$signatures and the 500 genes comprising the alcohol ones. Notably, for both sexes, the overlap was highly statistically significant (Supplemental Table S2). Next, we tested if there is an association between the direction (up/down) of the "response" to Adnp haploinsufficiency and alcohol exposure. While no interaction was found between the expression profiles of the 5month-old male/female $A d n p^{+/-}$mice and the alcohol exposed mice, we observed significant associations for the 1-month-old mice. Interestingly, while the $A d n p^{+/-}$male mice and chronic alcohol-exposure signatures showed significant positive association (Fig. 6a; Pearson's chi square: $X^{2}(1)=12.13, p<0.001$ ), the expression profile of the 1-month-old $A_{d n p^{+/-}}$female mice was negatively associated with the chronic alcohol-exposure signature (Fig. 6). Thus in 1-month-old females, greater overlap was found between the incongruent signatures in comparison to the congruent signatures (Fig. $5 \mathrm{~b} ; X_{(1)}^{2}=7.49, p<0.01$ ). Moreover, while the chronic-alcohol exposure down signature had an overlap of 21 genes with the male $A d n p^{+-}$down signature, and 23 genes with the females $A d n p^{+/-}$up signature, the intersection of these three signatures combined, provided 16 common genes (see genes marked in bold in Supplemental Table S3). For example, one of these common genes was transcription factor AP2 beta (TFAP2b), which is downregulated by chronic alcohol exposure [41]. This gene had a 3.8-fold increased expression in female $A d n p^{+/-}$mice, and in contrast, a five-fold decreased expression in male $\mathrm{Adnp}^{+/-}$mice, as compared to sex-matched Adnp $^{+/+}$controls.

GO enrichment analysis applied on each set of overlapping genes identified the functional category of "sequence-specific
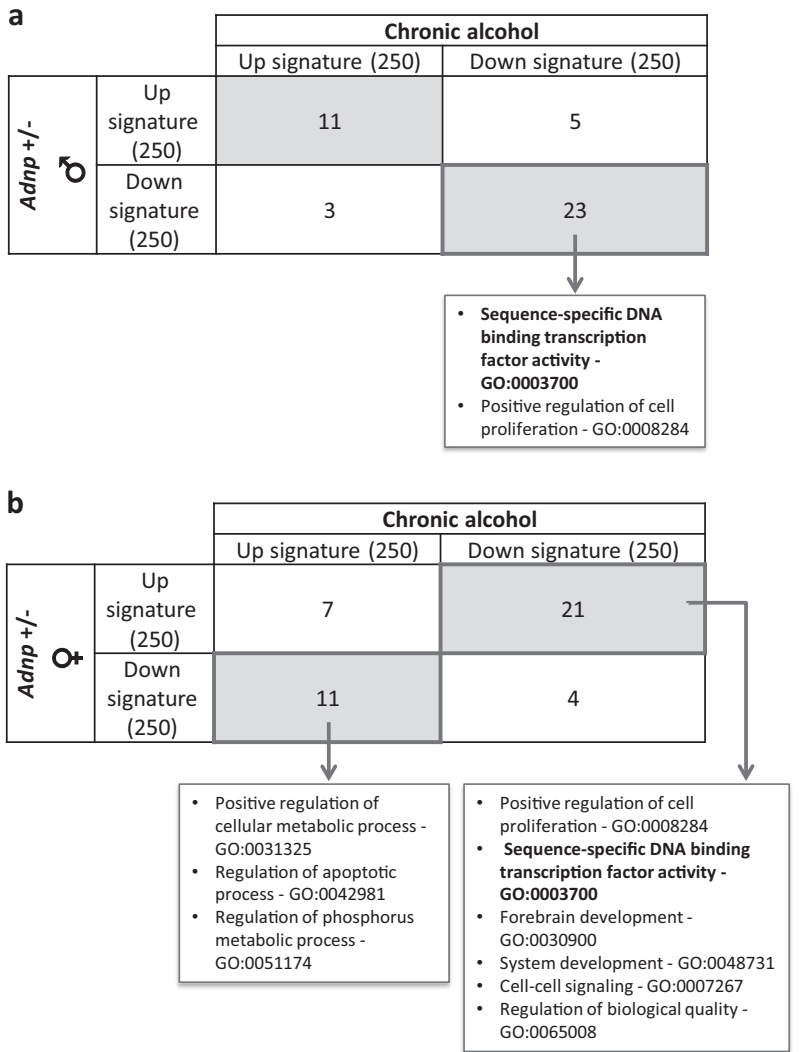

Fig. 6 Hippocampal alcohol-associated genes show opposing expression alterations in 1-month old male and female Adnp ${ }^{+/-}$ mice. Gene sets of the top 250 up-regulated genes ("Up-signature") and the top 250 down-regulated genes ("Down-signature") in alcohol-exposed adult male mice were intersected with the corresponding signatures of naïve male (a) and female (b) $A d n p^{+/-}$ mice. Numbers refer to the overlapping genes found in each comparison between a pair of signatures. Each group of overlapping genes was subjected to gene ontology (GO) functional enrichment analysis using the TANGO algorithm in Expander. The three groups of genes found to be enriched with functional categories $(p<0.05)$ are marked with bold framing 
DNA binding transcription factor activity" as enriched, in the gene set emerging from the comparison between the chronic-alcohol down signature and the male $A d n p^{+/-}$down signature, as well as from the comparison between the same chronic-alcohol signature with the female $A d n p^{+/-}$up signature.

\section{DISCUSSION}

We introduce here Adnp as a novel regulator of alcohol consumption in female mice. We show that while short-term alcohol administration increases Adnp expression in mesolimbic brain regions, prolonged voluntary consumption of excessive alcohol levels leads to sex-dependent alterations in the hippocampal expression of the gene transcript. We further show that reduced levels of the Adnp transcript lead to increased alcohol intake and preference selectively in female mice, which are reversed by the ADNP-derived neuroprotective peptide NAP. Together, our results suggest that ADNP plays a protective role against alcohol-drinking behavior.

\section{Adnp is an alcohol-responsive gene}

We focused on the mesolimbic system, which projects from the VTA of the midbrain to the NAc, hippocampus, and other limbic regions. The mesolimbic system has been implicated in processing of reward, including alcohol reward, and in addiction [11, 49]. Within the hippocampus, we focused on the dorsal part, which shows high Adnp expression in mice [50].

We found that Adnp mRNA expression was increased in the dorsal hippocampus of male and female mice, and in the NAc of females, following a repeated alcohol injection regimen (7 days), $24 \mathrm{~h}$ after the last injection. In contrast, prolonged, voluntary consumption of alcohol for 5 weeks resulted in a sustained decrease in Adnp expression in the dorsal hippocampus of female mice, and a transient increase in this mRNA in the dorsal hippocampus of male mice. Importantly, we have previously shown that the levels of Adnp mRNA correlate with, and reflect the ADNP protein levels $[51,52]$. It is important to note, that the experiments on alcohol-mediated regulation of Adnp were done on a C57BL/6J mice, which drink high levels of alcohol. However, due to their low-drinking levels, we did not test whether alcohol regulates Adnp expression on the mixed background of the Adnp haploinsufficient mouse model.

Previous studies measured Adnp transcript expression in mouse embryos (C57BI/6J) exposed to an ethanol bolus on day 8 of gestation, and found an increase in the Adnp transcripts $24 \mathrm{~h}$ after alcohol exposure, which persisted for 10 days [26]. Moreover, pups of dams that consumed alcohol during pregnancy were shown to have reduced Adnp mRNA levels compared to controls in the cerebral cortex at post-natal day 7 [27]. Together, these results emphasize the temporal control of Adnp expression, also within the context that ADNP acting as a transcription factor downregulates its own expression $[17,53]$. Here, we show that alcohol consumption leads to altered Adnp expression in the mesolimbic system of the adult mouse, further discovering alcohol-related sex-specific changes.

Reduced Adnp gene dosage lead to increased alcohol consumption in female mice, which is reversed by NAP We found that female $A d n p^{+/-}$mice displayed higher levels of alcohol consumption and preference, compared to $A d n p^{+/+}$mice. We used a drinking protocol of escalating alcohol concentrations, which generates a dose-response curve of alcohol intake as a function of its concentration. We found that female Adnp haploinsufficient mice showed increased alcohol intake in all concentrations tested, providing an upward shift of the dose-response curve, compared to control $\mathrm{Adnp}^{+/+}$females. This shift is considered to reflect an effect on the motivation to selfadminister alcohol [54-56], suggesting that reduced ADNP levels increases the motivation to consume alcohol. In fact, Adnp ${ }^{+/-}$ females showed a sharp increase in alcohol intake along with the increase in alcohol concentration, whereas $A d n p^{+/+}$mice showed only a moderate increase. Thus, ADNP seems to reduce the motivation to consume alcohol. Finally, we found that the consumption of a non-drug reinforcer, saccharin, or of a bitter taste (quinine solution) was not affected by Adnp haploinsufficiency in female mice, suggesting that the effect of Adnp was unique to alcohol reinforcement.

Critically, we found that daily treatment with NAP, prevented the increased alcohol intake in Adnp haploinsufficient female mice. NAP is an ADNP-derived peptide that was previously shown to protect against ADNP deficiency $[25,57]$. Thus, we expected that NAP administration to $A d n p^{+/-}$females would compensate for the reduced ADNP levels, and presumably normalize the phenotype. Indeed, in the 2 weeks of NAP treatment, we observed a complete reversal of the enhanced alcohol intake in $\mathrm{Adnp}^{+/-}$, confirming that reduced ADNP levels mediated the increased alcohol intake phenotype in the Adnp haploinsufficient female mice.

Interestingly, NAP has been previously shown to protect against alcohol toxicity in the fetal alcohol syndrome, associated with changes in ADNP expression [26, 58]. Mechanistically, alcohol inhibits neuronal differentiation/plasticity by disrupting ADNP signaling, which in turn is protected by NAP treatment [59]. Here, we observed reduction of Adnp expression by excessive alcohol consumption in the female mouse hippocampus, and in a separate experiment we found that $A d n p^{+-}$females show increased alcohol intake, reversed by NAP. It would be interesting to investigate in the future, whether the developmental alcohol-ADNP-NAP interactions share similar mechanisms to their interactions in adulthood.

The sex-dependent effects we found, i.e., the observation that increased alcohol intake and preference in $\mathrm{Adnp}^{+/-}$mice emerged only among females, indicate that the function of ADNP as regulator of alcohol drinking, may be differentially manifested in females and males. Indeed, Gozes and colleagues have found sex differences in ADNP expression in whole hippocampal sample from 5-month to 6-month old Adnp ${ }^{+/+}$mice, as well as in human hippocampal samples [19], which are extended to multiple differential gene regulation [14,60] (also see Fig. 6 and Supplemental Table S3). Furthermore, ADNP was shown to be regulated during the estrus cycle in the arcuate nucleus of the mouse [51].

Interestingly, alcohol induces ligand-independent activation of the estrogen receptor alpha [61]. Estrogen regulates multiple genes, including the sexually dichotomous ADNP regulator, vasoactive intestinal peptide (VIP) $[39,51,62,63]$. The VIP gene has a CAMP/CREB responsive element [64], and in turn, increases CAMP production in the target cells [65], bringing another connection to the CREB regulatory protein and alcohol consumption [11].

In order to better understand the sex-dependent regulatory mechanisms underlying ADNP-associated alcohol consumption, we used bioinformatics analyses with published hippocampal databases [14, 41]. Our analysis revealed that a substantial portion of the genes that demonstrate altered expression as a result of chronic alcohol exposure, behave in an opposite manner in 1month-old male and female mice with Adnp haploinsufficiency, in line with our sex-dependent results. No correlations of the $\mathrm{Adnp}^{+/-}$signature with alcohol exposure were obtained while measuring hippocampal gene expression at 5 months, in contrast to one month of age, suggesting a strong developmental impact. Indeed, GO functional categories, such as "Forebrain development" and "System development" were enriched in the common genes set of the female $A d n p^{+/-}$up-signature and the alcohol exposure down-signature (Fig. 6b). This association indicates that alcohol exposure and Adnp haploinsufficiency may lead to opposite changes in the expression of genes that play a functional 
role in forebrain and system development, which may be related to the tendency of $A d n p^{+/-}$females to consume more alcohol. It is important to note, that alcohol reduced Adnp expression in adult female C57BL/6 mice, whereas we did not test its effects in 1month old pups. Thus, taken together with our findings in wildtype C57BL/6 mice, the age-dependent findings raise the possibility that the ADNP-dependent regulatory mechanisms may be different, depending on whether Adnp expression is decreased from birth (as in the Adnp haploinsufficiency model), or whether its expression is decreased after alcohol exposure during adulthood.

As indicated in the results (Supplemental Table S3), using published RNA-seq data [14], we calculated here that the transcription factor Tfap $2 b$ had a 3.8-fold increased expression in female $\mathrm{Adnp}^{+/-}$, and in vast contrast, a five-fold decreased expression in $A d n p^{+/-}$male compared to $A d n p^{+/+}$controls. Critically, TFAP2b polymorphism has been implicated in alcoholism in women, whereby the high-functioning allele of the gene was more common among female alcoholic patients, compared to the non-alcoholic controls [66]. This finding resembles the increased alcohol consumption we observed in $\mathrm{Adnp}^{+/-}$females, that have increased Tfap $2 b$ expression.

Our finding that male mice consumed significantly lower levels of alcohol compared to females is in line with numerous previous studies (e.g., [67-70]). The genetic background of the mice we used (outbreeding of the Adnp transgenic line with the ICR strain) $[14,19]$, led to a relatively low baseline alcohol consumption and preference in both sexes, allowing the demonstration of increases in these alcohol-drinking measures. This very low baseline levels of alcohol consumed by both $A d n p^{+/+}$and $A d n p^{+/-}$male mice, due to their genetic background, may have masked possible effects of low ADNP levels on alcohol consumption in males, limiting our conclusions to female mice. However, the absence of genotype effect in males was not likely related to low baseline alcohol consumption, as Adnp haploinsufficiency increased, rather than decreased consumption.

We show in a model of moderate continuous drinking (Fig. 3) that Adnp haploinsufficiency leads to increased alcohol intake in females. However, in a model of heavy intermittent drinking (IA2BC, consisting of cycles of 24-h alcohol drinking and deprivation sessions), Adnp ${ }^{+/+}$control females increased drinking, to a level similar to that of $A d n p^{+/-}$female mice, resulting in the absence of a genotype effect. Here, a longer alcohol-deprivation period (12 days) was required for the genotype effect to emerge (Fig. 5). Interestingly, unlike $A d n p^{+/+}$controls, $A d n p^{+/-}$female mice consumed similar alcohol quantities in both the alcohol (20\%)-drinking protocols we used here: the continuous-alcohol access (Fig. 3), which typically leads to lower alcohol-drinking levels [34]; and the IA2BC (Fig. 5), which typically leads to higher drinking levels [34]. This finding further suggests that Adnp haploinsufficient females mice tend to develop increased drinking phenotype, even in conditions that do not yield heavy drinking in normal controls. Together with the finding that increased alcohol drinking in Adnp haploinsufficient females was reversed by NAP (Fig. 4), these results emphasize the relations between alcohol consumption and the Adnp genotype, as further illustrated in Fig. 6.

Abstinence periods can generate in rodents an "alcohol deprivation effect" [71, 72], i.e., consumption of considerably higher amounts of alcohol after a period of abstinence. However, this effect is usually seen in rodents drinking high amount of alcohol for several months [72], although it may appear sooner in some mouse strains (e.g., [71]). In our experiment, baseline alcohol consumption was relatively low, and mice were submitted to abstinence after only 5 weeks of drinking. We did not observe an increase in alcohol consumption in $\mathrm{Adnp}^{+/+}$controls, similar to previous reports with other mouse strains under limited-access to alcohol conditions [31]. However, we did observe the effect in the $A d n p^{+/-}$females, suggesting that normal Adnp expression and function gates the alcohol deprivation effect, at least in females. Thus, this finding indicates that Adnp haploinsufficiency leads to increased relapse to alcohol drinking after abstinence, suggesting that Adnp regulates relapse behavior in females

In conclusion, our findings suggest that at the initial exposure to alcohol, the mesolimbic expression of Adnp is increased, possibly to protect against the harmful effects of alcohol. However, this protective mechanism selectively breaks in females (that consume more alcohol in rodents compared to males), and Adnp expression is downregulated in the dorsal hippocampus, leading to escalation of alcohol drinking and sharp relapse after abstinence. Administration of the ADNP-derived peptide NAP reversed the effects of Adnp haploinsufficiency on alcohol consumption, presumably by compensating for deficient ADNP levels. Thus, it is possible that ADNP is a biomarker for alcohol-drinking behaviors, and that restoration of the function of ADNP in females may prevent drinking escalations, hence providing a novel therapeutic target to explore.

\section{ACKNOWLEDGEMENTS}

We thank Jasmine Heruty, Meitar Grad, Tanya Lenchinski, Yossi Sadot-Sogrin, and the Eshkol Fellows Gal Hacohen Kleiman, Shlomo Sragovich and Gidon Karmon for their technical assistance and input. Professor Illana Gozes is the incumbent of the Lily and Avraham Gildor Chair for the Investigation of Growth Factors and heads the Dr. Diana and Ziga (Zelman) Elton (Elbaum) Laboratory for Molecular Neuroendocrinology at Tel Aviv University.

\section{AUTHOR CONTRIBUTIONS}

$I G, Y Z$, and $S B$ designed the research; $Y Z, N R, O E-C, N L, E G, A M$, and OS performed the research; $Y Z, N R, O E-C, R E$, IG, and SB analyzed data, $Y Z, N R, N L, I G$, and SB wrote the paper.

\section{FUNDING}

The research was supported by a grant from the Dr. Miriam and Sheldon G. Adelson Center for the Biology of Addictive Diseases (IG \& SB). YZ was partly supported by a scholarship from the Dr. Miriam and Sheldon G. Adelson Graduate School of the Faculty of Medicine at Tel Aviv University (IG) and this paper is in partial fulfillment of his M.Sc. requirements. The research was further supported by funds from the Israel Science Foundation (ISF) grants 968-13 and 1916-13 (SB), and the German Israeli Foundation (GIF) grant I-2348-105.4/2014 (SB). Additional grants (IG) included the ISF grant (1424/14), ERA-NET neuron AUTYSM, AMN Foundation, Drs. Ronith and Armand Stemmer and Mr Arthur Gerbi (French Friends of Tel Aviv University), as well as Canadian and Spanish Friends of Tel Aviv University.

\section{ADDITIONAL INFORMATION}

Supplementary Information accompanies this paper at (https://doi.org/10.1038/ s41386-018-0132-7)

Competing interests: . NAP (also known as davunetide, CP201), under patent protection (IG), is being developed, under agreement with Ramot at Tel Aviv University, by Coronis Neurosciences for the ADNP syndrome (IG Chief Scientific Officer).

Publisher's note: Springer Nature remains neutral with regard to jurisdictional claims in published maps and institutional affiliations.

\section{REFERENCES}

1. Koob GF, Volkow ND. Neurocircuitry of addiction. Neuropsychopharmacology. 2009;35:217-38

2. Spanagel R. Alcoholism: a systems approach from molecular physiology to addictive behavior. Physiol Rev. 2009;89:649-705.

3. Ben Hamida S, Neasta J, Lasek AW, Kharazia V, Zou M, Carnicella S, et al. The small $\mathrm{G}$ protein $\mathrm{H}$-Ras in the mesolimbic system is a molecular gateway to alcoholseeking and excessive drinking behaviors. J Neurosci. 2012;32:15849-58. 
4. Neasta J, Ben Hamida S, Yowell Q, Carnicella S, Ron D. Role for mammalian target of rapamycin complex 1 signaling in neuroadaptations underlying alcoholrelated disorders. Proc Natl Acad Sci USA. 2010;107:20093-8.

5. Neasta J, Ben Hamida S, Yowell QV, Carnicella S, Ron D. AKT signaling pathway in the nucleus accumbens mediates excessive alcohol drinking behaviors. Biol Psychiatry. 2011;70:575-82.

6. Yao L, Arolfo MP, Dohrman DP, Jiang Z, Fan P, Fuchs S, et al. betagamma Dimers mediate synergy of dopamine D2 and adenosine A2 receptor-stimulated PKA signaling and regulate ethanol consumption. Cell. 2002;109:733-43.

7. Even-Chen O, Sadot-Sogrin Y, Shaham O, Barak S. Fibroblast growth factor 2 in the dorsomedial striatum is a novel positive regulator of alcohol consumption. J Neurosci. 2017;37:8742-54.

8. Ahmadiantehrani S, Barak S, Ron D. GDNF is a novel ethanol-responsive gene in the VTA: implications for the development and persistence of excessive drinking. Addict Biol. 2014;19:623-33.

9. Logrip ML, Barak S, Warnault V, Ron D. Corticostriatal BDNF and alcohol addiction. Brain Res. 2015;1628:60-7.

10. Barak S, Ahmadiantehrani S, Logrip ML, Ron D. GDNF and alcohol use disorder. Addict Biol. 2018. https://doi.org/10.1111/adb.12628.

11. Ron D, Barak S. Molecular mechanisms underlying alcohol-drinking behaviours. Nat Rev Neurosci. 2016;17:576-91.

12. Nestler EJ. Molecular neurobiology of addiction. Am J Addict/Am Acad Psychiatr Alcohol Addict. 2001;10:201-17.

13. Pinhasov A, Mandel S, Torchinsky A, Giladi E, Pittel Z, Goldsweig AM, et al Activity-dependent neuroprotective protein: a novel gene essential for brain formation. Brain Res Dev Brain Res. 2003;144:83-90.

14. Amram N, Hacohen-Kleiman G, Sragovich S, Malishkevich A, Katz J, Touloumi O, et al. Sexual divergence in microtubule function: the novel intranasal microtubule targeting SKIP normalizes axonal transport and enhances memory. Mol Psychiatry. 2016;21:1467-76.

15. Oz S, Kapitansky O, Ivashco-Pachima Y, Malishkevich A, Giladi E, Skalka N, et al. The NAP motif of activity-dependent neuroprotective protein (ADNP) regulates dendritic spines through microtubule end binding proteins. Mol Psychiatry. 2014;19:1115-24

16. Mandel S, Gozes I. Activity-dependent neuroprotective protein constitutes a novel element in the SWI/SNF chromatin remodeling complex. J Biol Chem. 2007;282:34448-56.

17. Mandel S, Rechavi G, Gozes I. Activity-dependent neuroprotective protein (ADNP) differentially interacts with chromatin to regulate genes essential for embryogenesis. Dev Biol. 2007;303:814-24.

18. Zamostiano R, Pinhasov A, Gelber E, Steingart RA, Seroussi E, Giladi E, et al Cloning and characterization of the human activity-dependent neuroprotective protein. J Biol Chem. 2001;276:708-14.

19. Malishkevich A, Amram N, Hacohen-Kleiman G, Magen I, Giladi E, Gozes I. Activity-dependent neuroprotective protein (ADNP) exhibits striking sexual dichotomy impacting on autistic and Alzheimer's pathologies. Transl Psychiatry 2015;5: e501.

20. Malishkevich A, Marshall GA, Schultz AP, Sperling RA, Aharon-Peretz J, Gozes I. Blood-borne activity-dependent neuroprotective protein (ADNP) is correlated with premorbid intelligence, clinical stage, and Alzheimer's disease biomarkers. J Alzheimers Dis. 2016:50:249-60.

21. Dresner E, Agam G, Gozes I. Activity-dependent neuroprotective protein (ADNP) expression level is correlated with the expression of the sister protein ADNP2: deregulation in schizophrenia. Eur Neuropsychopharmacol. 2011;21:355-61.

22. Merenlender-Wagner A, Malishkevich A, Shemer Z, Udawela M, Gibbons A, Scar $E$, et al. Autophagy has a key role in the pathophysiology of schizophrenia. Mol Psychiatry. 2015;20:126-32.

23. Helsmoortel C, Vulto-van Silfhout AT, Coe BP, Vandeweyer G, Rooms L, van den Ende J, et al. A SWI/SNF-related autism syndrome caused by de novo mutations in ADNP. Nat Genet. 2014;46:380-4.

24. Van Dijck A, Vulto-van Silfhout AT, Cappuyns E, van der Werf IM, Mancini GM Tzschach A, et al. Clinical presentation of a complex neurodevelopmental disorder caused by mutations in ADNP. Biol Psychiatry. 2018. https://doi.org/ 10.1016/j.biopsych.2018.02.1173.

25. Vulih-Shultzman I, Pinhasov A, Mandel S, Grigoriadis N, Touloumi O, Pittel Z, et al. Activity-dependent neuroprotective protein snippet NAP reduces tau hyperphosphorylation and enhances learning in a novel transgenic mouse model. J Pharmacol Exp Ther. 2007;323:438-49.

26. Poggi SH, Goodwin K, Hill JM, Brenneman DE, Tendi E, Schinelli S, et al. The role of activity-dependent neuroprotective protein in a mouse model of fetal alcohol syndrome. Am J Obstet Gynecol. 2003;189:790-3.

27. Pascual M, Guerri C. The peptide NAP promotes neuronal growth and differentiation through extracellular signal-regulated protein kinase and Akt pathways, and protects neurons co-cultured with astrocytes damaged by ethanol. J Neurochem. 2007;103:557-68.
28. Farris SP, Arasappan D, Hunicke-Smith S, Harris RA, Mayfield RD. Transcriptome organization for chronic alcohol abuse in human brain. Mol Psychiatry. 2015;20:1438-47.

29. Zipori D, Sadot-Sogrin Y, Goltseker K, Even-Chen O, Rahamim N, Shaham O, et al. Re-exposure to nicotine-associated context from adolescence enhances alcohol intake in adulthood. Sci Rep. 2017;7:2479.

30. Ben Hamida S, Darcq E, Wang J, Wu S, Phamluong K, Kharazia V, et al. Protein tyrosine phosphatase alpha in the dorsomedial striatum promotes excessive ethanol-drinking behaviors. J Neurosci. 2013;33:14369-78.

31. Carnicella S, Ahmadiantehrani S, Janak PH, Ron D. GDNF is an endogenous negative regulator of ethanol-mediated reward and of ethanol consumption after a period of abstinence. Alcohol Clin Exp Res. 2009;33:1012-24.

32. Hwa LS, Debold JF, Miczek KA. Alcohol in excess: CRF(1) receptors in the rat and mouse VTA and DRN. Psychopharmacology (Berl). 2013;225:313-27.

33. Warnault V, Darcq E, Levine A, Barak S, Ron D. Chromatin remodeling-a novel strategy to control excessive alcohol drinking. Transl Psychiatry. 2013;3:e231.

34. Hwa LS, Chu A, Levinson SA, Kayyali TM, DeBold JF, Miczek KA. Persistent escalation of alcohol drinking in C57BL/6J mice with intermittent access to $20 \%$ ethanol. Alcohol Clin Exp Res. 2011;35:1938-47.

35. Barak S, Wang J, Ahmadiantehrani S, Ben Hamida S, Kells AP, Forsayeth J, et al Glial cell line-derived neurotrophic factor (GDNF) is an endogenous protector in the mesolimbic system against excessive alcohol consumption and relapse. Addict Biol. 2015;20:629-42.

36. Alcalay RN, Giladi E, Pick CG, Gozes I. Intranasal administration of NAP, a neuroprotective peptide, decreases anxiety-like behavior in aging mice in the elevated plus maze. Neurosci Lett. 2004;361:128-31.

37. Morimoto BH, De Lannoy I, Fox AW, Gozes I, Stewart A. Davunetide pharmacokinetics and distribution to brain after intravenous or intranasal administration to rat. Chimica oggi 2009; 27.

38. Gozes I. Chapter 13 - Neuroprotective drug development: the story of ADNP, NAP (Davunetide), and SKIP. In: Gozes I (ed). Neuroprotection in Alzheimer's Disease. 1st Edition, Academic Press, Elsevier. 2017;253-70.

39. Bassan M, Zamostiano R, Davidson A, Pinhasov A, Giladi E, Perl O, et al. Complete sequence of a novel protein containing a femtomolar-activity-dependent neuroprotective peptide. J Neurochem. 1999;72:1283-93.

40. Gozes I, Giladi E, Pinhasov A, Bardea A, Brenneman DE. Activity-dependent neurotrophic factor: intranasal administration of femtomolar-acting peptides improve performance in a water maze. J Pharmacol Exp Ther. 2000;293:1091-8.

41. Smith ML, Lopez MF, Archer KJ, Wolen AR, Becker HC, Miles MF. Time-course analysis of brain regional expression network responses to chronic intermittent ethanol and withdrawal: implications for mechanisms underlying excessive ethanol consumption. PLoS ONE. 2016;11:e0146257.

42. Ulitsky I, Maron-Katz A, Shavit S, Sagir D, Linhart C, Elkon R, et al. Expander: from expression microarrays to networks and functions. Nat Protoc. 2010;5: 303-22.

43. Griffin WC 3rd. Alcohol dependence and free-choice drinking in mice. Alcohol. 2014;48:287-93.

44. Gozes I, Morimoto BH, Tiong J, Fox A, Sutherland K, Dangoor D, et al. NAP: research and development of a peptide derived from activity-dependent neuroprotective protein (ADNP). CNS Drug Rev. 2005;11:353-68.

45. Gozes I, Ivashko-Pachima Y, Sayas CL. ADNP, a microtubule interacting protein provides neuroprotection through end binding proteins and tau: an amplifier effect. Front Mol Neurosci. 2018;11:151.

46. Ivashko-Pachima $\mathrm{Y}$, Sayas $\mathrm{CL}$, Malishkevich A, Gozes I. ADNP/NAP dramatically increase microtubule end-binding protein-Tau interaction: a novel avenue for protection against tauopathy. Mol Psychiatry. 2017;22:1335-44.

47. Wang $\mathrm{K}$, Song $\mathrm{H}$, Jin $\mathrm{M}$, Xiao $\mathrm{H}$, Zhao $\mathrm{G}$, Zou H, et al. Chronic alcohol consumption from adolescence to adulthood in mice-hypothalamic gene expression changes in insulin-signaling pathway. Alcohol. 2014;48:571-8.

48. Zou H, Xie Q, Zhang M, Zhang C, Zhao G, Jin M, et al. Chronic alcohol consumption from adolescence-to-adulthood in mice-effect on growth and social behavior. Drug Alcohol Depend. 2009;104:119-25.

49. Koob GF, Volkow ND. Neurobiology of addiction: a neurocircuitry analysis. Lancet Psychiatry. 2016;3:760-73.

50. Allen Institute for Brain Science. Allen Mouse Brain Atlas. 2004. http://mouse. brain-map.org. 1 October 2018.

51. Furman S, Hill JM, Vulih I, Zaltzman R, Hauser JM, Brenneman DE, et al. Sexual dimorphism of activity-dependent neuroprotective protein in the mouse arcuate nucleus. Neurosci Lett. 2005;373:73-8.

52. Schirer Y, Malishkevich A, Ophir Y, Lewis J, Giladi E, Gozes I. Novel marker for the onset of frontotemporal dementia: early increase in activity-dependent neuroprotective protein (ADNP) in the face of Tau mutation. PLoS ONE. 2014;9:e87383.

53. Aboonq MS, Vasiliou SA, Haddley K, Quinn JP, Bubb VJ. Activity-dependent neuroprotective protein modulates its own gene expression. J Mol Neurosci. 2012;46:33-9. 
424

54. Carnicella S, Yowell QV, Ron D. Regulation of operant oral ethanol self-administration: a dose-response curve study in rats. Alcohol Clin Exp Res. 2011;35:116-25.

55. Ahmed SH, Koob GF. Long-lasting increase in the set point for cocaine selfadministration after escalation in rats. Psychopharmacology (Berlin). 1999;146:303-12.

56. Zernig G, Ahmed SH, Cardinal RN, Morgan D, Acquas E, Foltin RW, et al. Explaining the escalation of drug use in substance dependence: models and appropriate animal laboratory tests. Pharmacology. 2007;80:65-119.

57. Gozes I, Divinski I, Piltzer I. NAP and D-SAL: neuroprotection against the beta amyloid peptide (1-42). BMC Neurosci. 2008;9:S3.

58. Spong CY, Abebe DT, Gozes I, Brenneman DE, Hill JM. Prevention of fetal demise and growth restriction in a mouse model of fetal alcohol syndrome. J Pharmacol Exp Ther. 2001;297:774-9.

59. Chen S, Charness ME., Ethanol inhibits neuronal differentiation by disrupting activity-dependent neuroprotective protein signaling. Proc Natl Acad Sci USA. 2008;105:19962-19967.

60. Gozes I. Sexual divergence in activity-dependent neuroprotective protein impacting autism, schizophrenia, and Alzheimer's disease. J Neurosci Res. 2017:95:652-60.

61. Etique N, Flament S, Lecomte J, Grillier-Vuissoz I. Ethanol-induced ligand-independent activation of ERalpha mediated by cyclic AMP/PKA signaling pathway: an in vitro study on MCF-7 breast cancer cells. Int J Oncol. 2007;31:1509-18.

62. Gozes I, Werner H, Fawzi M, Abdelatty A, Shani Y, Fridkin M, et al. Estrogen regulation of vasoactive intestinal peptide mRNA in rat hypothalamus. J Mol Neurosci. 1989;1:55-61.
63. Rostene W, Montagne MN, Dussaillant M, Gozes II. Steroid regulation of vasoactive intestinal peptide (VIP). Stress. 1997;2:79-90.

64. Giladi E, Shani Y, Gozes I. The complete structure of the rat VIP gene. Brain Res Mol Brain Res. 1990;7:261-7.

65. Gozes I, McCune SK, Jacobson L, Warren D, Moody TW, Fridkin M, et al. An antagonist to vasoactive intestinal peptide affects cellular functions in the central nervous system. J Pharmacol Exp Ther. 1991;257:959-66.

66. Nordquist N, Gokturk C, Comasco E, Nilsson KW, Oreland L, Hallman J. Transcription factor AP2 beta involved in severe female alcoholism. Brain Res. 2009;1305:S20-6.

67. Jury NJ, DiBerto JF, Kash TL, Holmes A. Sex differences in the behavioral sequelae of chronic ethanol exposure. Alcohol. 2017;58:53-60.

68. Lancaster FE, Spiegel KS. Sex differences in pattern of drinking. Alcohol. 1992;9:415-20.

69. Meliska CJ, Bartke A, McGlacken G, Jensen RA. Ethanol, nicotine, amphetamine, and aspartame consumption and preferences in C57BL/6 and DBA/2 mice. Pharmacol Biochem Behav. 1995;50:619-26.

70. Tambour S, Brown LL, Crabbe JC. Gender and age at drinking onset affect voluntary alcohol consumption but neither the alcohol deprivation effect nor the response to stress in mice. Alcohol Clin Exp Res. 2008;32:2100-6.

71. Khisti RT, Wolstenholme J, Shelton KL, Miles MF. Characterization of the ethanoldeprivation effect in substrains of C57BL/6 mice. Alcohol. 2006:40:119-26.

72. Vengeliene V, Bilbao A, Spanagel R. The alcohol deprivation effect model for studying relapse behavior: a comparison between rats and mice. Alcohol. 2014;48:313-20. 